\title{
8 A Modelagem Matemática na formação continuada de professores \\ o relato de uma experiência
}

Carlos Roberto Ferreira

\section{SciELO Books / SciELO Livros / SciELO Libros}

FERREIRA, C. R. A Modelagem Matemática na formação continuada de professores: o relato de uma experiência. In: BRANDT, C. F., BURAK, D., and KLÜBER, T. E., orgs. Modelagem matemática: perspectivas, experiências, reflexões e teorizações [online]. 2nd ed. rev. and enl. Ponta Grossa: Editora UEPG, 2016, pp. 147-162. ISBN 978-85-7798-232-5. Available from: doi: 10.7476/9788577982325.0009. Also available in ePUB from: http://books.scielo.org/id/b4zpq/epub/brandt-9788577982325.epub.

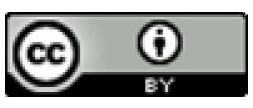

All the contents of this work, except where otherwise noted, is licensed under a Creative Commons Attribution $\underline{4.0 \text { International license. }}$

Todo o conteúdo deste trabalho, exceto quando houver ressalva, é publicado sob a licença $\underline{\text { Creative Commons }}$ Atribição 4.0.

Todo el contenido de esta obra, excepto donde se indique lo contrario, está bajo licencia de la licencia $\underline{\text { Creative }}$ Commons Reconocimento 4.0. 


\title{
A Modelagem Matemática na formação continuada de professores: o relato de uma experiência
}

\author{
Carlos Roberto Ferreira
}

\section{Introdução}

O acontecimento relatado a seguir, referente a uma experiência envolvendo Modelagem Matemática, ocorreu em 1990 em um curso de pósgraduação em Educação Matemática. Contudo, antes de relatar a experiência propriamente dita, apresento, na sequência, um breve histórico dos acontecimentos que a antecederam.

Era um dos formandos em Matemática, da turma de 1987, da Faculdade de Filosofia, Ciências e Letras de Arapongas, e o momento era de alegria para todos os presentes, com sentimento de missão cumprida. E, enquanto aguardava a entrega do diploma, sentia-me angustiado com pensamentos do presente e do futuro. Como já possuía alguma experiência como profissional da educação, pois ensinava matemática há dois anos para uma turma de $8^{\mathrm{a}}$ série, comecei a fazer uma retrospectiva da minha prática como professor de matemática, dos resultados alcançados e, com isso, uma "ponta" de preocupação começava a se fazer presente. Na semana que antecedeu a formatura, houve um conselho de classe em que, de uma das turmas, de um total de 40 estudantes, 10 ficaram reprovados na minha disciplina. No momento não me preocupei porque ouvi dos colegas vários comentários: "não se preocupe, isso é normal"; "matemática é assim mesmo, muito difícil"; "com essa nota tem que reprovar mesmo"; "se não prestar bem atenção e não fizer as listas de exercícios não se aprende matemática"; "este aluno vai bem nas disciplinas da área de humanas, mas nas disciplinas de exatas é um desastre, ele não sabe tabuada, não tem raciocínio"; entre outros.

Algo me chamou a atenção, o semblante de um colega professor com mais tempo de magistério. Ocorreram 13 reprovações em matemática na sua turma e ele parecia orgulhoso e realizado, como se aquelas reprovações fossem mesmo o seu objetivo. Sorridente, aparentava superioridade frente aos outros professores cujas disciplinas apenas 2 ou 3 estudantes ficaram reprovados.

Os meus educandos reprovados, os comentários dos professores e a reação do meu colega professor de matemática, fizeram-me refletir e trouxeram-me vários questionamentos, como: a culpa pelas reprovações era 
somente dos estudantes ou a metodologia utilizada poderia ter contribuído? São os educandos que não aprendem ou são os professores que não ensinam adequadamente? A metodologia que utilizava era apenas uma reprodução da prática dos meus professores ao longo da minha vida escolar? Será que era a correta? Os alunos que foram aprovados aprenderam Matemática ou apenas decoraram algoritmos e os repetiam de forma mecânica? Será que eles conseguiam atribuir significado e a utilizaram como instrumento para a vida em suas mais diversas situações? O material didático, com um breve resumo da teoria, uma fórmula e alguns exercícios resolvidos, foi adequado?

$\mathrm{Na}$ busca por respostas às questões mencionadas, teve início uma trajetória de leituras e discussões durante as reuniões pedagógicas da escola. Já nas leituras iniciais foi possível encontrar alguns dados importantes sobre o ensino de Matemática, nos quais os resultados de alguns levantamentos indicavam o baixo rendimento dos estudantes em matemática. Apontavam ainda o escasso conhecimento da disciplina pela população.

Com isso, já era possível perceber que o ensino de Matemática não estava alcançando os objetivos propostos: desenvolver a autonomia, resolver situações e/ou problemas do cotidiano, enfim, tornar o aluno um cidadão pleno. Vários problemas, dentre eles a formação dos professores, a pesquisa sobre novos métodos de ensino e a elaboração e desenvolvimento de materiais didáticos, mereciam atenção especial. Assim, a consciência da necessidade e da dificuldade de se construir o conhecimento matemático não era nova. Enfrentar a questão era imperativo!

Lidar com a Matemática enseja também o desenvolvimento da capacidade de abstração. Entendemos que o resultado desse desenvolvimento deve contribuir para a aplicação da matemática em diversas situações da vida do indivíduo. (BURAK, 1987; 1992; 1998; 2004). A vida em sociedade cobra um mínimo de conhecimento matemático, sem o qual, a própria cidadania do indivíduo fica comprometida. Empreender um negócio, acompanhar a evolução de uma campanha eleitoral, controlar o orçamento doméstico, verificar o rendimento de uma aplicação financeira, interpretar um gráfico de inflação, exige algum conhecimento matemático e a consciência de como ele influencia a sociedade. (SKOVSMOSE, 2007).

Os apontamentos feitos, até este momento, encaminham os assuntos tratados neste capítulo. As seções subsequentes contemplam uma descrição detalhada sobre o projeto de Modelagem Matemática desenvolvido no curso de formação de professores, a perspectiva de Modelagem adotada, o desenvolvimento do modelo que minimiza os custos para a implantação de uma 
rede de energia elétrica. A estrutura escolhida busca evidenciar como essa atividade influenciou significativamente na minha prática em sala de aula.

\section{A origem do projeto}

Em 1990 iniciei um curso de pós-graduação lato sensu promovido pela Fundação Faculdade Estadual de Filosofia, Ciências e Letras de Guarapuava, hoje Universidade Estadual do Centro-Oeste - UNICENTRO, que foi concluído em 1991. O curso teve como objetivo qualificar professores de Matemática em uma nova forma de conceber o ensino da Matemática. Essa nova forma se constituía na aplicação da Modelagem Matemática. É evidente que, naquele momento, ainda não se tinha clareza sobre as implicações do trabalho com a Modelagem, mas a iniciativa foi louvável e promissora, uma vez que se buscava romper com os moldes de ensino mais usuais.

O encaminhamento das atividades desenvolvidas no curso se deu da seguinte forma: a turma de professores participantes foi dividida em pequenos grupos e o trabalho foi realizado conforme as orientações acerca da Modelagem Matemática na época. O tema escolhido pelo grupo foi "A eletricidade como fator de integração social", pois, após uma visita às vilas da cidade, foi possível observar que várias delas ainda não possuíam eletricidade, causando todo tipo de transtorno aos seus moradores, como problemas de segurança, conservação de alimentos, lazer, entre outros.

Uma pesquisa foi elaborada, na qual as entrevistas com os moradores evidenciaram a baixa autoestima entre eles, por se sentirem excluídos do resto da população. Conhecendo-se a situação das vilas, buscamos ouvir as autoridades do município em relação aos resultados da pesquisa. Percebemos que o motivo principal para a não instalação da rede de energia elétrica nos bairros era a falta de recursos financeiros. Uma questão se fez presente: como minimizar os custos do projeto de eletrificação de uma das vilas para torná-lo viável?

Naquele momento não existiam muitas bibliografias disponíveis sobre Modelagem Matemática no ensino de Matemática, porém, muitos dos aspectos apresentados em publicações posteriores já se faziam presentes ao desenvolvermos os projetos e no curso ministrado. Por isso, a próxima seção aborda alguns dos elementos que consideramos importantes, para posteriormente passarmos à descrição da construção do modelo matemático. 


\section{Considerações sobre a Modelagem Matemática e a concepção assumida}

A preocupação maior não era estudar a Matemática pela Matemática, mas inseri-la em um contexto. No caso, para resolver um problema social relacionado à implantação da rede elétrica na vila. Essa ideia vinha ao encontro da Modelagem Matemática, pois, segundo Bassanezi (1994; 2002), ela é o estudo de problemas ou de situações-reais, que atua como uma linguagem utilizada para a compreensão, simplificação e resolução destas situações, visando uma possível previsão ou modificação do objeto estudado.

O problema escolhido pelo grupo ensejou a construção de um modelo matemático em conformidade com o que Bassanezi (1990; 2002) explicita, ou seja, o modelo matemático é quase sempre um sistema de equações ou inequações algébricas, diferenciais, integrais, que é obtido através do estabelecimento de relações entre as variáveis que são essenciais no fenômeno analisado. (BASSANEZI, 1990; 2002).

Segundo Jacobini (1999), outra característica importante da Modelagem no ensino relaciona-se com a necessidade de coleta de dados e da pesquisa sobre o assunto em estudo, sendo essas tarefas realizadas pelos estudantes e, em geral, em grupos.

Outro importante aspecto que naquele momento pôde ser observado foi a possibilidade de transformação social por meio dos conteúdos matemáticos adquiridos pelos sujeitos. Esse aspecto é enfatizado por Caldeira (2004), que também afirma que essa aprendizagem deve partir do contexto sociocultural do aluno, proporcionando-lhe o desenvolvimento do pensamento lógico, da criatividade, de aprender conceitos e de construir estruturas matemáticas, a fim de compreender a realidade social, histórica e cultural.

Mais especificamente sobre a concepção assumida nesse trabalho, que vem sendo construída ao longo de mais de duas décadas por Burak (1987, 1992, 1998, 2004 e 2006), podemos destacar que a Modelagem Matemática vem ao encontro das expectativas do educando, por dar sentido ao que ele estuda, por satisfazer suas necessidades de aprendizagem, partindo dos seus interesses, podendo realizar alguns de seus objetivos. Vale destacar que sua preocupação está centrada no processo de ensino e de aprendizagem da Matemática na Educação Básica. Fato esse que favorece a reconfiguração da modelagem como uma metodologia fundamentada em teorias de ensino e de aprendizagem e visão de ciência, aspectos que a diferenciam de outras perspectivas. Portanto, há 
um olhar intencional sobre os processos cognitivos da aprendizagem. Os pressupostos adotados, principalmente aquele que se refere ao interesse dos participantes, é oriundo da experiência de cunho antropológico e das teorias construtivistas, interacionistas e de aprendizagem significativa. (BURAK, 1998). Por esses motivos, existe a possibilidade de o aluno trabalhar com entusiasmo e perseverança, formando atitudes positivas em relação à matemática, ou seja, pode despertar nele o gosto pela disciplina.

O desenvolvimento de uma atividade de Modelagem Matemática, na perspectiva de Burak (1998 e 2004), sugere cinco etapas: 1) escolha do tema; 2) pesquisa exploratória; 3) levantamento dos problemas; 4) resolução dos problemas e desenvolvimento do conteúdo matemático no contexto do tema; 5) análise crítica das soluções. Essas etapas devem sempre ser encaminhadas levando-se em consideração os dois princípios propostos pelo autor: 1) o interesse do grupo e 2) a obtenção de informações e dados do ambiente, onde se encontra o interesse do grupo. Durante todo o processo da Modelagem a postura do professor é primordial, pois ele assume o papel de mediador, orientador e problematizador.

Em linhas gerais, é possível afirmar que o trabalho desenvolvido naquele momento seguiu os pressupostos dessa concepção de Modelagem, uma vez que esse pesquisador fazia parte do corpo docente da pós-graduação e influenciou significativamente as nossas atividades.

\section{Processo de construção do modelo}

Entre as diversas questões levantadas na elaboração do projeto - como por exemplo: qual o material a ser utilizado? Que quantidades? -, incluiu-se a da iluminação de ruas, objeto deste relato. O objetivo foi encontrar um modelo matemático que relacionasse a distância entre os postes (D), o iluminamento (i), a intensidade luminosa (I) e a altura do poste (h), conforme a Figura 1. Esse modelo deve indicar a distância mínima entre dois postes que proporcione uma claridade adequada, e assim determinar se é possível diminuir a quantidade de postes a serem utilizados e, consequentemente, os custos de eletrificação da vila. 
Figura 1 - Postes

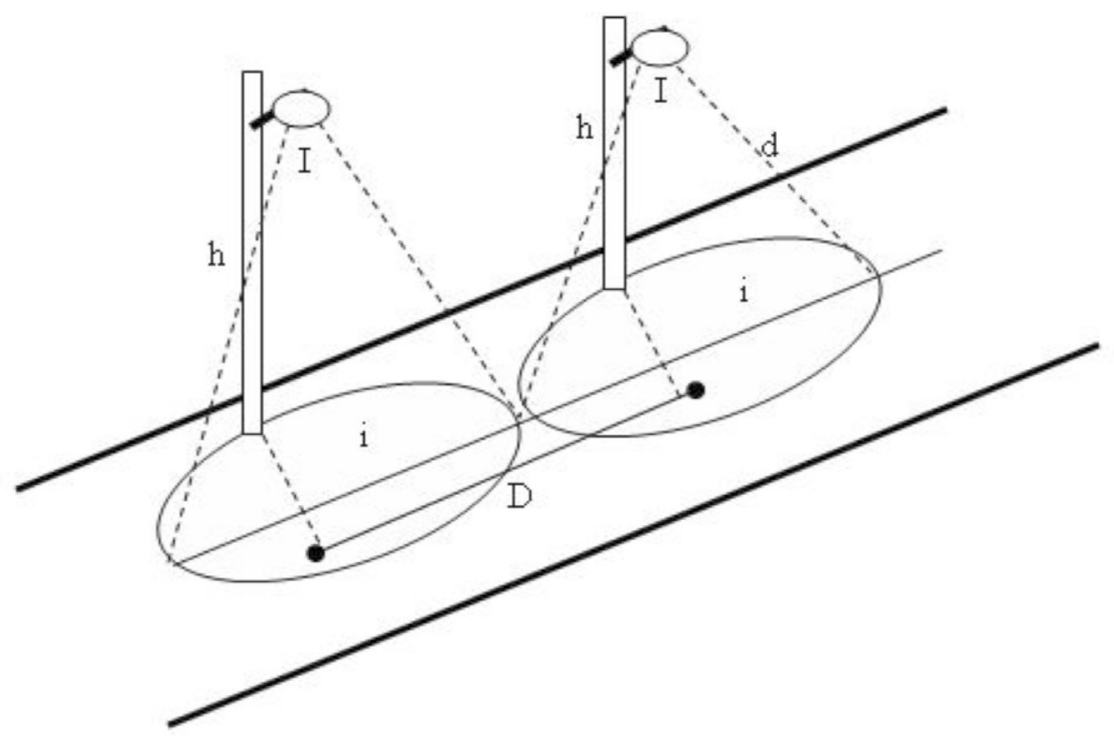

Na figura 1 temos:

$$
\begin{aligned}
& \text { I = intensidade luminosa; } \\
& \text { i = iluminamento; } \\
& \text { h = altura do poste; } \\
& \text { d = distância entre o ponto iluminado e a base da luminária; } \\
& \text { D = distância entre os postes. }
\end{aligned}
$$

\subsection{Conceitos de Física}

Para o desenvolvimento desse modelo, foi necessário fazer uma revisão na literatura de Física sobre o conteúdo de Fotometria, com conceitos como:

\subsubsection{Fluxo luminoso (F)}

O fluxo luminoso é a quantidade de energia produzida por uma fonte luminosa. A unidade de fluxo luminoso é chamada lúmen, que se define como o fluxo emitido por um foco puntiforme, com intensidade de uma candela, segundo um ângulo sólido de um esferorradiano. Percebemos bem essa definição, imaginando uma esfera de um metro de raio, tendo no centro o foco de uma candela. Se fizermos na superfície da esfera uma 
abertura de um metro quadrado, a quantidade de luz que passará por essa abertura será de um lúmen.

\subsubsection{Intensidade luminosa (I) - candela}

Intensidade luminosa de uma fonte em uma determinada direção é a razão entre o fluxo luminoso $(\mathrm{F})$, que ela emite através de um pequeno ângulo sólido $(\Omega)$ cujo eixo é a direção considerada, e esse ângulo sólido

$$
I=\frac{F}{\Omega} .
$$

\subsection{3 Ângulo sólido $(\Omega)$}

Considera-se uma superfície esférica qualquer, de raio $\mathrm{R}$, com centro no vértice do ângulo sólido, mede-se a área $\mathrm{S}$ da superfície esférica, subtendida pelo ângulo sólido, e obtém-se o valor do ângulo sólido, em esferorradianos, dividindo a área $\mathrm{S}$ pelo quadrado do raio, isto é

$$
\Omega=\frac{S}{R^{2}}
$$

\subsubsection{Fluxo luminoso total $(\mathrm{Ft})$}

De $I=\frac{F}{\Omega}$ tiramos $F=I . \Omega$. O fluxo total emitido pela fonte correspondente a um ângulo sólido máximo $\Omega_{\max }=4 \pi$ esferorradianos. Logo: $F=4 \pi$.I. Essa equação só pode ser usada se a intensidade luminosa da fonte for constante em todas as direções.

\subsubsection{Iluminamento (i) - Lux}

Iluminamento de uma superfície é a razão entre o fluxo luminoso (F), que sobre ela incide, e a sua área

$$
\text { (S): } i=\frac{F}{S} \text {. }
$$

Com essas definições claras, iniciamos a construção do modelo. 
O cálculo de iluminamento será feito em função da altura da luminária, mas poderia também ser feito em função da distância que o ponto iluminado está da base da luminária, conforme a Figura 2:

Figura 2 - Iluminamento

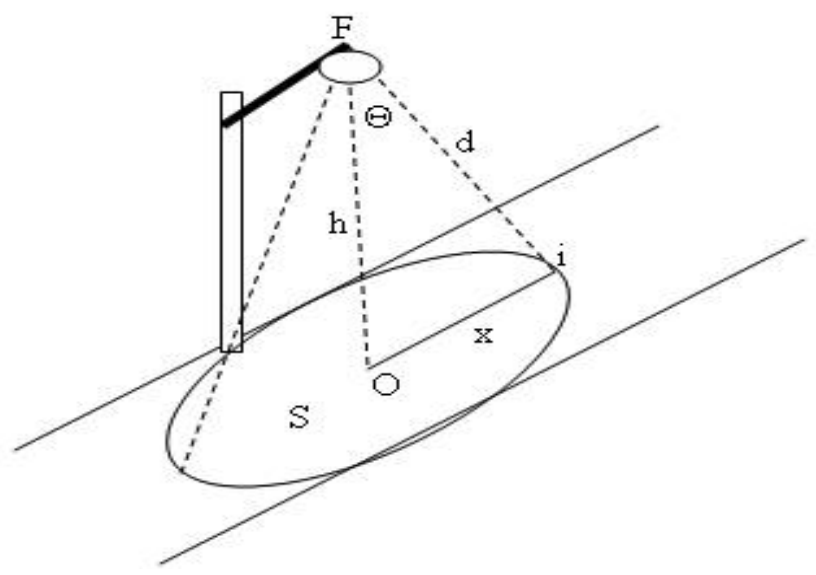

Com a ajuda de um fotômetro (aparelho utilizado para medir a iluminação em um ponto) medimos a iluminação no ponto $i$, para cada altura da luminária (Tabela 1). Foi considerada uma lâmpada de $125 \mathrm{w}$, com fluxo luminoso igual a 5.400 lumens (fornecido pelo fabricante) e de ângulo de incidência constante igual a $60^{\circ}$, teremos:

\begin{tabular}{c|c}
\hline \multicolumn{2}{c}{ VALORES DA ALTURA } \\
\hline \multicolumn{2}{c}{ E ILUMINAÇÃO } \\
\hline $\mathrm{h}$ & $\mathrm{i}$ \\
\hline 0,5 & 215 \\
\hline 1,0 & 53,75 \\
\hline 2,0 & 13,43 \\
\hline 3,0 & 5,97 \\
\hline 4,0 & 3,35 \\
\hline 5,0 & 2,25 \\
\hline \multicolumn{2}{|c}{ TABELA 1} \\
\hline
\end{tabular}

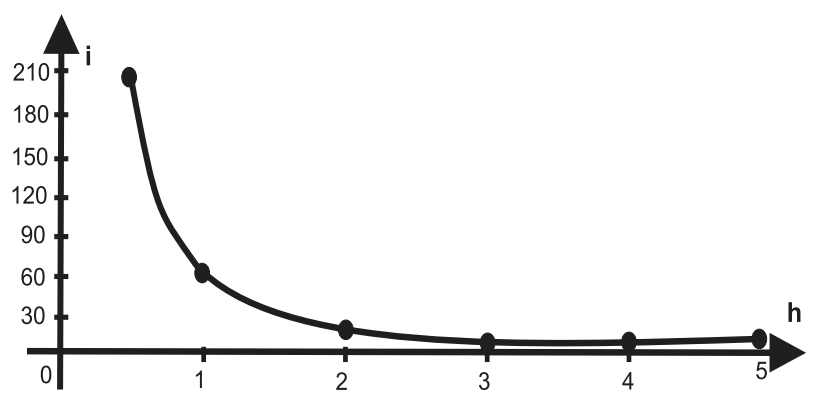

Pelo esboço do gráfico, já foi possível perceber que a curva é uma hipérbole e a função é do tipo potência. 


\subsection{Demonstrando:}

Como $\mathrm{i}=\mathrm{f}(\mathrm{h})$, temos duas características importantes sobre o gráfico:

a) se $h \rightarrow \infty$ então $i \rightarrow 0$;

b) se $i \rightarrow \infty$ então $h \rightarrow 0$.

Estas características representam uma função do tipo

$$
i=\frac{a}{g(h)}, \operatorname{com} g(h)=h \text { temos que } i=\frac{a}{h^{b}} \text {, sendo } a \text { e } b \text { constantes. }
$$

Esta função pode ser caracterizada como uma função racional.

Agora, o próximo passo é encontrar o valor da constante $\mathbf{a}$ e $\mathbf{b}$ através da Regressão Linear.

Como já sabemos $i=f(h)=\frac{a}{h^{b}}=a \cdot h^{-b}$ e calculando o logaritmo de ambos os membros temos:

$$
\ln i=\ln a-b \ln h
$$

Fazendo: $\left\{\begin{array}{l}\ln i=Y \\ \ln a=A \\ \ln h=H\end{array} \quad\right.$ temos $Y=A-b H \quad$ (Equação da Reta).

$\left.\mathbf{1}^{\circ}\right)$ A constante a pode ser calculada fazendo $\mathbf{h}=\mathbf{1}$. Substituindo

$h=1 \mathrm{em} i=\frac{a}{h^{b}}$, temos $i=a$. Observando a Tabela 1 , temos que para $h=1$ o valor para $i$ encontrado foi de $53,75 \operatorname{lux}^{1}$.

$\left.2^{\circ}\right) \mathrm{O}$ valor de $\mathbf{b}$ pode ser calculado com base nos valores encontrados e descritos na Tabela 1. Aplicando a fórmula $Y=A-b H$ podemos elaborar a Tabela 2 , na qual o valor de $\mathbf{b}$ pode ser calculado pela expressão $i=\frac{a}{h^{b}}$, pois os valores de $\mathbf{i}, \mathbf{a}$ e $\mathbf{h}$ já são conhecidos.

\footnotetext{
${ }^{1}$ Este valor foi medido utilizando uma lâmpada de $125 \mathrm{~W}$ de 5400 lumens e está registrado na Tabela 1.
} 
Tabela 2 - Determinação dos Valores de "a" e "b"

\begin{tabular}{|c|c|c|c|c|c|c|}
\hline $\mathbf{h}$ & $\mathbf{i}$ & $\mathbf{a}$ & $\mathbf{Y}=\mathbf{I n i}$ & $\mathbf{A}=\mathbf{l n a}$ & $\mathbf{H}=\mathbf{l n h}$ & $\mathbf{b}$ \\
\hline 0,5 & 215,00 & 53,75 & 5,37064 & 3,98434 & $-0,69315$ & 2 \\
\hline 1 & 53,75 & 53,75 & 3,98434 & 3,98434 & 0,00000 & $*$ \\
\hline 2 & 13,44 & 53,75 & 2,59824 & 3,98434 & 0,69315 & 2 \\
\hline 3 & 5,97 & 53,75 & 1,78675 & 3,98434 & 1,09861 & 2 \\
\hline 4 & 3,36 & 53,75 & 1,21194 & 3,98434 & 1,38629 & 2 \\
\hline 5 & 2,15 & 53,75 & 0,76547 & 3,98434 & 1,60944 & 2 \\
\hline 6 & 1,49 & 53,75 & 0,39878 & 3,98434 & 1,79176 & 2 \\
\hline 7 & 1,10 & 53,75 & 0,09531 & 3,98434 & 1,94591 & 2 \\
\hline 8 & 0,84 & 53,75 & $-0,17435$ & 3,98434 & 2,07944 & 2 \\
\hline 9 & 0,66 & 53,75 & $-0,41552$ & 3,98434 & 2,19722 & 2 \\
\hline 10 & 0,54 & 53,75 & $-0,61619$ & 3,98434 & 2,30259 & 2 \\
\hline
\end{tabular}

Nesse ponto surgiu uma questão interessante, para $h=1$ temos $H=$ 0 , ou seja, não é possível obter o valor de $b$ utilizando a fórmula $Y=A-b H$. Poderíamos apenas informar que o valor de $b$ não está definido para $h=1$, mas também poderíamos aproveitar a oportunidade para introduzir o conceito de Limites de Função, pois para $h=1$ o valor de $b$ não está definido, mas para valores próximos de 1 pela direita ou pela esquerda o valor de $b$ fica próximo de 2.

Assim, a equação para cálculos de iluminamento de áreas abertas será:

$$
i=\frac{a}{h^{2}}
$$

Na Figura 2, $S$ é a superfície iluminada pela fonte pontual $F$. O eixo $O F$ do ângulo sólido $\Omega$, determinado por $F$ e $S$, encontra a superfície no ponto $O$. A superfície esférica $S$, subtendida pelo ângulo sólido $\Omega$, foi traçada com centro em $F$ e raio $O F=d$.

Estamos considerando um ângulo sólido suficientemente pequeno para que se possa confundir a superfície esférica $S$ ' com uma superfície plana. Sendo assim, $S$ e $S^{\prime}$ formam entre si o mesmo ângulo $\Theta$, que é o ângulo de incidência.

Podemos então escrever que: $S^{\prime}=S \cdot \cos \Theta$. 
Como o ângulo sólido $\Omega$ é definido por: $\Omega=\frac{S^{\complement}}{d^{2}}$, temos:

$$
\Omega=\frac{S \cdot \cos \Theta}{d^{2}}
$$

De $i=\frac{F}{S}$ e $I=\frac{F}{\Omega}$, definições de iluminamento e intensidade luminosa, chegamos a:

$$
\begin{aligned}
& F=i . S \\
& F=I . \Omega \\
& i S=I \Omega
\end{aligned}
$$

Substituindo pelo seu valor, dado pela equação (1), obtemos:

$$
\begin{gathered}
i S=I \frac{S \cdot \cos \Theta}{d^{2}} \\
i=\frac{I \cdot \cos \Theta}{d^{2}}
\end{gathered}
$$

\subsubsection{Fórmula de Lambert}

Após construído esse modelo, algo fascinante aconteceu. Descobrimos que o modelo já existia e é conhecido como Fórmula de Lambert. Esse fato trouxe uma satisfação muito grande, experimentamos a (re)descoberta. Esse é um dos aspectos que consideramos de muita importância na formação dos professores, pois a oportunidade de construção nos foi dada, saímos de um modelo de ensino, de apenas repetir e receber informações prontas. Nesse sentido, acreditamos que essa experiência pode, em suas devidas proporções, ser efetivada também na Educação Básica, haja vista acreditarmos que quando os professores têm oportunidades diferenciadas como estudantes podem efetivá-las também na sua prática docente. A nossa experiência corrobora essa afirmação, pois a partir desse momento iniciamos um processo de mudança de atitude como professor de Matemática. 
Quanto ao modelo, não se havia chegado ao resultado esperado e ainda não estava pronto, pois a fórmula a ser empregada, no caso da iluminação de áreas abertas, deveria ser a que permitisse determinar o iluminamento produzido por uma luminária, em cada ponto de uma dada superfície horizontal.

Assim, considera-se a Figura 3, onde $F$ é um foco luminoso, com $I$ candelas de intensidade, iluminado um ponto $A$. Calcula-se os iluminamentos $i$, produzidos por $F$, respectivamente, em um elemento de superfície horizontal e em um elemento de superfície vertical, situados ambos em $A$.

\section{Figura 3}

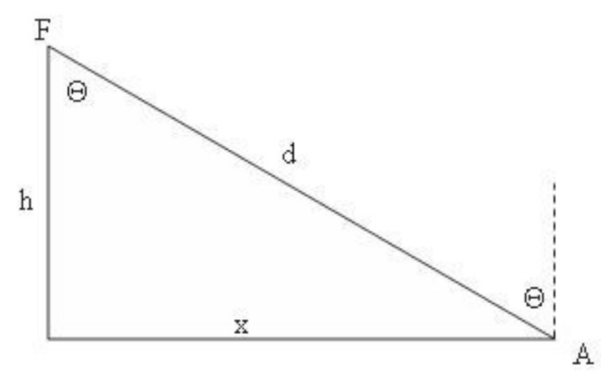

Pela figura, sendo $h$ a altura do foco $F$ acima do plano horizontal do ponto $A$, temos que: $d=\frac{h}{\cos \theta}$ e, portanto, $d^{2}=\frac{h^{2}}{\cos ^{2} \theta}$. Substituindo no modelo encontrado (2) $i=\frac{I \cdot \cos \theta}{d^{2}}$, encontramos: $i=\frac{I \cdot \cos ^{3} \theta}{h^{2}}$ (iluminamento em função da altura da luminária)

\subsubsection{Cálculo de $i$ em função de $x$}

Trata-se aqui do problema da iluminação de ruas e estradas, onde os focos são dispostos ao longo da área a iluminar, segundo uma única fila de postes.

Conforme a Figura 3, $x$ é a distância do ponto de menor ilumina-mento $(0,1$ lux $)$, e o ponto $O$. 
Fazendo $\operatorname{tg} \theta=\frac{x}{h}$, temos que $\quad h=\frac{x}{\operatorname{tg} \theta} \quad$ e substituído em $i=\frac{I \cdot \cos ^{3} \theta}{h^{2}}$ encontramos:

$$
i=\frac{I \cdot \cos ^{3} \theta \cdot \operatorname{tg}^{2} \theta}{x^{2}}
$$

Assim, isolando $x$, chegamos ao seguinte modelo:

$$
x=\sqrt{\frac{I \cdot \cos ^{3} \theta \cdot \operatorname{tg}^{2} \theta}{i}}
$$

\subsubsection{O resultado encontrado pelo modelo}

Determinação da Intensidade luminosa $(I)$ :

$$
\begin{aligned}
& F_{t}=4 \pi I \\
& 5400=4.3,14 . I \\
& I=430 \text { candelas }
\end{aligned}
$$

Passaremos agora para o cálculo da distância em que se encontra o menor iluminamento considerado, que seria de 0,1 lux até o centro.

Para um iluminamento de 0,5lux o ângulo deverá ser de:

$$
i=\frac{I \cdot \cos ^{3} \theta}{h^{2}} \therefore \cos ^{3} \theta=\frac{i \cdot h^{2}}{I} \therefore \cos \theta=\sqrt[3]{\frac{i \cdot h^{2}}{I}} \therefore \cos \theta=\sqrt[3]{\frac{0,7^{2}}{430}} \therefore \theta=77^{\circ}
$$


Cálculo de $x$ :

$$
\begin{aligned}
& x=\sqrt{\frac{430 \cdot \cos ^{3} 77^{\circ} \cdot \operatorname{tg}^{2} 77^{\circ}}{0,5}}=\sqrt{\frac{430 \times 0,0113831 \times 18,7616}{0,5}}=\sqrt{1918,33} \\
& x=30
\end{aligned}
$$

Como a distância $D$ entre dois postes é dada por $D=2 x$ (Figura 1$)$, temos:

$D=30 \times 2=60$ metros para uma lâmpada de $125 \mathrm{~W}$. Considerando que as empresas de energia instalam os postes com uma distância média de 45 metros, podemos concluir que haveria uma economia razoável na quantidade de postes. Considerando que a vila tenha $10 \mathrm{~km}$ (10.000 metros) de ruas, dividindo por $45 \mathrm{~m}$ a empresa necessitará em torno de 222 postes, agora se utilizar a distância entre postes de 60 metros, irá utilizar em torno de 166 postes, uma economia de $25 \%$. Importante ressaltar que neste estudo não foi levado em consideração a tração no fio e sua catenária (curva gerada por um fio suspenso pelas suas extremidades e sujeita à ação da gravidade), estamos supondo que a passagem de $45 \mathrm{~m}$ de distância para $60 \mathrm{~m}$ não irá causar qualquer problema ao fio. Mas fica aqui a sugestão para o estudo da tração no fio e sua catenária.

\section{Considerações sobre a experiência}

Neste relato a construção do modelo estava inserida em um contexto, ou seja, nasceu de uma necessidade de soluções que proporcionassem a minimização dos custos do projeto de eletrificação de uma vila na cidade de Guarapuava, Paraná. É importante ressaltar que além da construção do modelo, que consideramos fundamental, temos também que valorizar o processo reflexivo de construção. O envolvimento dos professores participantes com a realidade social, para a resolução do problema é considerado por nós um fator primordial, pois com isso a visão de uma formação estanque, apenas focada em conteúdos matemáticos, cai por terra.

Há a necessidade de enfrentamento de outras realidades. Essa condição pode favorecer o reconhecimento por parte dos professores, das dificuldades que os estudantes enfrentam e de, alguma maneira, humanizar o ensino de Matemática, que tantas vezes prioriza apenas o intelectual sem levar em conta outras dimensionalidades da vida humana, tais como os fatores econômicos, sociais, religiosos, entre outros. 
Aspectos interdisciplinares puderam ser percebidos durante a investigação e resolução dos problemas vinculados ao tema escolhido, principalmente em relação aos estudos dos conceitos de Física, pois, sem eles, não seria possível a resolução do problema e o enfrentamento do fenômeno estudado. Aspectos transdisciplinares envolvendo a situação econômica do país, a questão da segurança e até mesmo da saúde das pessoas, também foram constatados e esse é mais um ponto que consideramos importante, haja vista que incitou atitudes de diálogo nas aulas de Matemática, a qual tende a fechar-se em suas próprias estruturas. Tal diálogo facilita uma atitude interdisciplinar e transdisciplinar em relação ao conhecimento em questão.

Nesta experiência foi possível perceber que aprender Matemática não significa receber todos os conceitos prontos. Os conceitos devem ser construídos com base nos que foram construídos anteriormente. Nessa perspectiva, segundo Bassanezi (1994 e 2002), os estudantes podem generalizar, estruturar ou desestruturar o universo matemático, para que possam compreender e resolver as situações-problema, que podem ser de natureza matemática ou originadas na realidade de cada indivíduo.

Não se tratou aqui de estudar teorias ou técnicas de resolução de modelos matemáticos, pois essas podem ser memorizadas, aprendidas e esquecidas; este relato procurou mostrar que houve um desafio conceitual e um raciocínio lógico e crítico que são, por sua vez, essenciais ao processo da Modelagem Matemática no ensino e aprendizagem da Matemática.

\section{Referências}

BASSANEZI, R. C. A Modelagem como estratégia de ensinoaprendizagem. Campinas: Unicamp, 1990. jun, 1994.

Modelagem matemática. Dynamics, Blumenau, v. 1, n. 7, abr/

. Ensino-aprendizagem com modelagem matemática: uma nova estratégia. São Paulo: Contexto, 2002.

BURAK, D. Modelagem matemática: uma alternativa para o ensino de matemática na $5^{a}$ série. 1987. Dissertação (Mestrado em Educação Matemática) - Universidade Estadual Paulista, Rio Claro, 1987.

. Modelagem matemática: ações e interações no processo de ensinoaprendizagem. Tese (Doutorado em Educação) - Universidade Estadual de Campinas, Campinas, 1992. 
. Formação dos pensamentos algébricos e geométricos: uma experiência com modelagem matemática. Pró-Mat. - Paraná. Curitiba, v.1, n.1, p.32-41, 1998.

. A modelagem matemática e a sala de aula. In: Encontro Paranaense de Modelagem em Educação Matemática, 1, 2004, Londrina. Anais... Londrina: UEL, 2004. p. 1-10.

. Modelagem matemática: avanços, problemas e desafios. In: Encontro Paranaense de Modelagem em Educação Matemática, 2, 2006, Apucarana, PR. Anais... Modelagem Matemática: Práticas, Críticas e Perspectivas de Modelagem na Educação Matemática: Apucarana: FAP, 2006. p. 1-9.

CALDEIRA, A. D. Modelagem matemática e a prática dos professores do ensino fundamental e médio. In: Encontro Paranaense de Modelagem em Educação Matemática, 1, 2004, Londrina. Anais... Londrina: UEL, 2004.

JACOBINI, O. A modelação matemática aplicada no ensino de estatística em cursos de graduação. Dissertação de Mestrado. UNESP, Rio Claro. 1999.

SKOVSMOSE, O. Educação crítica: incerteza, matemática, responsabilidade. Trad. Maria Aparecida Viggiani Bicudo. São Paulo: Cortez, 2007. 